\title{
Article \\ FPGA Realization of the Parameter-Switching Method in the Chen Oscillator and Application in Image Transmission
}

\author{
Vincent-Ademola Adeyemi ${ }^{1,+}\left(\mathbb{D}\right.$, Jose-Cruz Nuñez-Perez ${ }^{1, *,+} \oplus$, Yuma Sandoval Ibarra ${ }^{2,+} \oplus$, \\ Francisco-Javier Perez-Pinal ${ }^{3,+}$ (D) and Esteban Tlelo-Cuautle ${ }^{4,+}$ \\ 1 Instituto Politécnico Nacional, IPN-CITEDI, Tijuana 22435, Mexico; vademola@citedi.mx \\ 2 Departamento de Posgrado, Universidad Politécnica de Lázaro Cárdenas (UPLC), Lázaro Cárdenas 60950, \\ Mexico; yumasandoval@uplc.edu.mx \\ 3 Tecnológico Nacional de México, Instituto Tecnológico de Celaya, Celaya 38010, Mexico; \\ francisco.perez@itcelaya.edu.mx \\ 4 Instituto Nacional de Astrofísica, Óptica y Electrónica, INAOE, San Andres Cholula 72840, Mexico; \\ etlelo@inaoep.mx \\ * Correspondence: jnunez@ipn.mx; Tel.: +52-55-5729-6000 \\ + These authors contributed equally to this work.
}

check for

updates

Citation: Adeyemi, V.-A.;

Nuñez-Perez, J.-C.

Sandoval Ibarra, Y.; Perez-Pinal, F.-J.;

Tlelo-Cuautle, E. FPGA Realization of

the Parameter-Switching Method in

the Chen Oscillator and Application

in Image Transmission. Symmetry

2021, 13, 923. https://doi.org/

$10.3390 /$ sym 13060923

Academic Editor: Wiesław Leonski

Received: 9 April 2021

Accepted: 17 May 2021

Published: 22 May 2021

Publisher's Note: MDPI stays neutral with regard to jurisdictional claims in published maps and institutional affiliations.

Copyright: (C) 2021 by the authors. Licensee MDPI, Basel, Switzerland. This article is an open access article distributed under the terms and conditions of the Creative Commons Attribution (CC BY) license (https:/ / creativecommons.org/licenses/by/ $4.0 /)$.

\begin{abstract}
In this paper, the parameter-switching technique was applied to control chaos in the Chen oscillator and as a decryption mechanism in a secure transmission system, to transmit RGB and grayscale images. In the past few decades, considerable efforts have been put into the study of the stabilization of chaotic dynamical systems. Most of the well-known chaos control methods, such as Ott, Grebogi, and Yorke (OGY), Pyragas, and open-loop methods, force an unstable periodic orbit into a stable one while distorting the original attractor. On the other hand, the parameterswitching technique is an elegant method that can synthesize an already-existing stable orbit, thereby preserving the underlying attractor. Consequently, the main contributions of this work were the FPGA realizations of the parameter-switching method and a secure image transmission system using a synchronized master and slave topology. The results of the parameter-switching technique and synchronization were verified using phase plots and time series. The chaos-encrypted image from the image transmission system, verified using correlation, showed no relativity with the original image, while the recovery of the decrypted image has no loss of quality. The encryption and decryption system was symmetric, whereby the key was private. In this work, co-simulations were performed in Active-HDL with MATLAB/Simulink, while the target FPGA board was the Xilinx's Artix-7 AC701.
\end{abstract}

Keywords: chaos; Chen oscillator; FPGA; image transmission; parameter switching; VHDL

\section{Introduction}

The concept and qualitative theory of dynamical systems was originated by a French mathematician, Henri Poincaré. The methods he developed prepared the foundation for the analysis of a nonlinear system of differential equations. Generally, in a nonlinear system, the time evolution of the states is controlled by laws that are dependent on the values of the state variables in a way that does not accomplish proportionality [1]. A nonlinear system consists of a state space, whose coordinates describe the state at any instant. To determine the state of a dynamical system in the long term, the system must be iterated several times, advancing in each iteration with a small step size. Starting with an initial point, the entire future positions of a nonlinear system can be known, provided the system has a solution. The future positions are collectively called a trajectory [2-5].

The characteristics of a nonlinear system can be verified by evaluating the bifurcation diagram, nonlinear divergence, multistability, fractal dimension, and limit cycle [6,7]. Bifurcation is defined as the change in the dynamical behavior of a nonlinear system while varying a parameter of the system. Nonlinear divergence occurs when the associated 
linear components of certain state variables are totally stable if the states approach infinity. Multistability is a nonlinear behavior defining the occurrence of more than two stable states. The fractal characteristic is seen when the system shows self-similarity in the phase space geometric pattern, whereby the pattern is reproduced at smaller sizes to generate surfaces and irregular shapes, which is impossible to show by conventional geometry. The limit cycle is a behavior whereby the nonlinear dynamical system possesses a significant periodic orbit. Lastly, an irregular and chaotic behavior occurs when the system displays sensitivity to the initial value.

The intrinsic behavior of nonlinear dynamical systems after a long time when illustrated by the phase space diagram is dependent on the basin of attraction [8-11]. The basin of attraction is simply the various initial conditions that give a dynamical system a particular qualitative state in the long term. Such qualitative behavior, in terms of the attractor, includes chaotic, periodic, or quasi-periodic. This, by implication, means that there exist in a nonlinear dynamical system different basins of attraction. Moreover, the inherent nonlinearity in the coupled system as a factor for the system instability cannot be overemphasized. The tuning of nonlinearities to enhance the dynamical behavior of the system, otherwise called functionalization, was studied in [12,13]. It has been shown that many physical phenomena are intrinsically nonlinear in nature. For instance, nonlinear models govern physical phenomena such as fluid and plasma mechanics, elasticity, aircraft and spacecraft control, gravitational and electrostatic attraction, gas dynamics, robotics, biological systems, bio-mechanics, combustion, and chemical reactions. The chaotic behavior of a nonlinear dynamical system is a basic property that encounters a huge number of applications in engineering. Specifically, nonlinear systems are fundamental to the concept of chaos theory, and deterministic and discrete chaotic dynamical systems are having many interventions in engineering and science [14-19].

As a parameter of a dynamical system is varied, chaos is generated from an infinite series of period-doubling bifurcations of periodic orbits. This is called the period doubling to chaos [20]. Chaotic attractors usually have closely-packed unstable periodic orbits (UPOs) embedded in them. In the past few years, considerable efforts, both theoretical and experimental, have been devoted to how to stabilize chaotic dynamical systems, whereby the orbits change a little under small perturbations of a parameter. This is otherwise called chaos control. Three famous chaos control techniques are the Ott, Grebogi, and Yorke (OGY) method, the Pyragas method, and the open-loop control method. The OGY is a discontinuous feedback control method, which works by first selecting an UPO from the chaotic system and, then, an appropriate parameter giving a small, wisely chosen perturbation once per cycle to keep the system on the selected orbit, while shifting and distorting the chaotic attractor to reach the stabilization of the general UPOs [21-23]. The Pyragas method is a feedback control technique, which applies an appropriate continuous control signal to the system through a feedback function [24-26]. In the case of the open-loop method, otherwise called the feed-forward method, a properly chosen external harmonic excitation is applied to the system to ensure the convergence of the trajectories to the desired periodic orbit $[27,28]$. Alternatively, a notable technique that is being used in controlling chaos in dynamical systems is the parameter-switching method [29-31]. Compared with the traditional chaos control techniques mentioned above, in which an UPO is forced into a stable periodic orbit, the parameter-switching method synthesizes an already existing stable orbit while preserving the underlying chaotic attractor.

Experimental verifications of chaotic systems through electronic implementations led us to create real applications based on chaos, such as: secure communication systems and cryptography. The field-programmable gate array (FPGA) is a viable option for digitally implementing chaotic systems. They have become a very dependable choice for the electronic implementation of chaotic systems because they can be reprogrammed, making the FPGAs reusable, adaptable, and extremely cost effective; hence, they have become the preferred option for the purpose of fast prototyping. Furthermore, FPGAs 
perform parallel processing at a higher speed. Moreover, designing FPGAs using hardware description languages (HDLs) is done very quickly and efficiently.

Much work have been done on the implementation of chaotic systems on FPGAs. For example: The authors in [32] showed a chaotic oscillator that was spherical in shape, realized on the Artix-7 AC701 and Stratix III boards for transmission of RGB and grayscale images. The numerical solution was obtained by applying the fifth Runge-Kutta method. Both their MATLAB and VHDL simulations were in good agreement. In [33], a Lü-Chen chaotic system was developed in VHDL with the Heun algorithm, implemented on a Virtex6 board, and the outputs confirmed the results obtained from MATLAB. Furthermore, the work in [34] showed a 3D chaotic system realized on an FPGA DE2-115 Altera board, using the Euler algorithm. The work included an application for encrypting an image with chaos. In [35], a novel chaotic TRNG that was based on an ANN was implemented on a Virtex- 6 board. In this work, the numerical method for the underlying chaotic oscillator was the fifth Runge-Kutta. A coupled map lattice, capable of displaying very complex spatiotemporal chaotic behavior, was used in [36] to create a cryptosystem and implemented on a Spartan-3 board. The article [37] considered the realization of a novel chaotic system, having one parameter, on a Cyclone IV board using forward Euler to find the solution. The investigation also included a secure communication system realized on the FPGA board. Other recent related works on the FPGA implementation of secure communication systems based on chaos can be found in [38-44].

In addition to the FPGA implementations discussed above, there are numerous numerical implementations of image security systems as seen in [45-48]. In the work presented in [45], a new image encryption system was proposed, based on a hyperchaotic oscillator, cellular automata, and particle swarm optimization. The authors in [46] presented a novel single-dimensional chaotic system and applied it to secure grayscale and RGB images. In the encryption process, bits were first shifted dynamically, and then, a diffusion method was applied to complete the image encryption. The work in [47] also applied a plain image to generate the keys to control the method of image encryption and compression, based on compressive sensing. The permutation and diffusion were performed by a $2 \mathrm{D}$ sine logistic iterative chaotic map and a 2D logistic sine coupling map. Finally, a novel approach was proposed in [48] to improve the cryptographic characteristics of substitution boxes, with the Choquet fuzzy integral and DNA methods as the backbone. The image encryption was performed using the DNA-coded fuzzy-based S-boxes and a Chen hyperchaotic map's chaotic sequence encoded by DNA. The numerical implementations of these works were performed in MATLAB.

In this work, the authors investigated the application of the parameter-switching technique in approximating the stable dynamics of the Chen oscillator and its usefulness in a chaos-based image transmission system, with a view of a digital implementation on an FPGA device. The encryption and decryption system embedded in the transmission system was based on the symmetric cryptographic concept in which the key was kept private. In light of the foregoing aim of this work, the following contributions to the state-of-the-art are hereby highlighted:

(i) FPGA realization of the parameter-switching scheme to approximate the stable cycles of the Chen oscillator, using VHDL as the implementation language with a word length of 24 bits, on the Xilinx's Artix-7 AC701 board. The VHDL implementation on the FPGA board agreed completely with the numerical simulations done in MATLAB;

(ii) FPGA realization of a secure chaos-based image transmission system on the Xilinx's Artix-7 AC701 board, using VHDL with a 24 bit word length, whereby the parameterswitching scheme was applied as a decryption mechanism to recover chaos-encrypted RGB and grayscale images. The backbone of the secure image transmission system was a synchronized master and slave Chen system, in which the state observer was the slave system that approximated the master system. The VHDL implementation and MATLAB numerical simulations of the image transmission were in complete agreement. 
In the subsequent parts of this paper, Section 2 introduces the mathematical model of the Chen oscillator, the parameter-switching technique applied in obtaining the stable cycles of the Chen oscillator, and the synchronization method. Section 3 presents the results of the synthesis of stable attractors by the parameter-switching scheme and synchronizing two Chen oscillators in a master and slave configuration. Section 4 details the secure image transmission system, with the application of the parameter-switching scheme for decrypting chaos-encrypted RGB and grayscale images. Section 5 contains the discussion of the results obtained in this work. Lastly, the conclusion is presented in Section 6.

\section{Theoretical Framework}

The Chen oscillator is one of the popular chaotic systems that has been widely researched in the past few years [49-52]. It is comprised of the following coupled system of ordinary differential equations of the first-order:

$$
\left\{\begin{array}{l}
\frac{d x}{d t}=a(y-x) \\
\frac{d y}{d t}=(c-a) x-x z+c y \\
\frac{d z}{d t}=x y-b z
\end{array}\right.
$$

where $x, y$, and $z$ are the system-dependent variables. Traditionally, the Chen oscillator is chaotic when $a=35, b=3$, and $c=28$. The oscillator is dissipative when $a+b>c$ and possesses the following three equilibrium points [49]: $E P_{0}=(0,0,0), E P_{1}=(\sqrt{b(2 c-a)}$, $\sqrt{b(2 c-a)}, 2 c-a)$, and $E P_{2}=(-\sqrt{b(2 c-a)},-\sqrt{b(2 c-a)}, 2 c-a)$. At $E P_{0}$, the eigenvalues are $\lambda_{1}=-3, \lambda_{2}=-30.8359$, and $\lambda_{3}=23.8359$. $E P_{1}$ and $E P_{2}$ have the same eigenvalues, which are $\lambda_{1}=-18.4280, \lambda_{2}=4.2140+14.8846 i$, and $\lambda_{3}=4.2140-14.8846 i$.

\subsection{Parameter-Switching Method}

The parameter switching (PS) is an elegant method for approximating numerically any desired solution of a continuous nonlinear and autonomous integer or fractionalorder dynamical system modeled by a general initial value problem (IVP) [29,30,53-55]. It involves selecting a limited number of parameter values in which the control parameter $p$ within the chosen set is switched in some periodic (deterministic) manner for relatively short time sub-intervals $i_{j}$, while numerically integrating the underlying IVP. At the end, the solution obtained from the "switched" system in the PS scheme will converge to the "averaged" solution when $p$ is changed to $p *$, which is the average of the values that are switched. The next general IVP describes many dynamical systems, the Chen system in Equation (1) inclusive:

$$
\frac{d x}{d t}=f(x(t))+p A x(t), \quad x(0)=x_{0}, \quad t \in I=[0, T]
$$

where $f: \boldsymbol{R}^{n} \rightarrow \boldsymbol{R}^{n}$ is a Lipschitz continuous nonlinear function, $p \in \boldsymbol{R}$ is the switched parameter, $x_{0} \in \boldsymbol{R}^{n}$ represents the initial value, $T>0$, and $A \in L\left(\boldsymbol{R}^{n}\right)$ is a constant matrix. Modeling the Chen system in Equation (1) after the IVP in Equation (2) with parameter $c=p$ as the control parameter and giving $a$ and $b$ their conventional values, then:

$$
f(x)=\left(\begin{array}{c}
a(y-x) \\
-a x-x z \\
x y-b z
\end{array}\right)
$$

and:

$$
A=\left(\begin{array}{lll}
0 & 0 & 0 \\
1 & 0 & 0 \\
0 & 0 & 0
\end{array}\right)
$$


The PS is expressed in the scheme that follows:

$$
\left[m_{1} p_{1}, m_{2} p_{2}, \ldots, m_{N} p_{N}\right]
$$

where $p_{k}$ are the control parameters, i.e., $p$ in the general IVP above, $m_{k}$ represents the weights associated with each $p_{j}$, and $N(N>1)$ represents the total number of switched parameters. When the IVP is being integrated, starting with $m_{1}$ integration steps, then $p=p_{1}$. In the following $m_{2}$ sub-interval, $p=p_{2}$. The integration continues in this manner until the final $m_{N}$ sub-interval, whereby $p=p_{N}$. Following the same procedure, the PS algorithm repeats again until the integration time interval $I$ is covered. Mathematically, sub-interval $i_{k}=m_{k} h$. Figure 1 gives the illustration of the PS scheme.

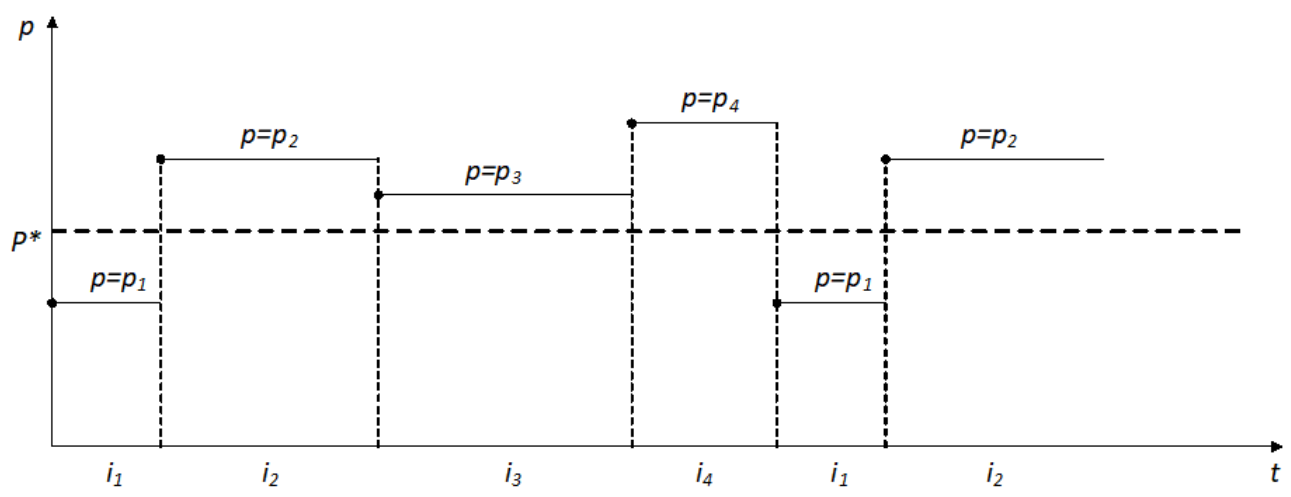

Figure 1. Parameter-switching scheme showing the switched parameters $p_{k}$ and their associated sub-interval $i_{k}$ times.

The "averaged" solution of $p^{*}$, to which the PS solution converges, is denoted by:

$$
p^{*}=\frac{\sum_{k=1}^{N} m_{k} p_{k}}{\sum_{k=1}^{N} m_{k}}
$$

Notation 1. Let $\mathcal{A}$ be the set of attractors depending on parameter $p, \mathcal{P}$ be the set of all possible $p$ values, and $\mathcal{M}$ be the set of all possible weights $m$.

Notation 2. $\mathcal{P}_{N}=\left\{p_{1}, p_{2}, \ldots, p_{N}\right\} \subset \mathcal{P}$ denotes the set of chosen $p . \mathcal{M}_{N}=\left\{m_{1}, m_{2}, \ldots, m_{N}\right\}$ $\subset \mathcal{M}$ is the set of chosen $m . \mathcal{A}_{N}=\left\{A^{p_{1}}, A^{p_{2}}, \ldots, A^{p_{N}}\right\} \subset \mathcal{A}$ is the set of the underlying attractors corresponding to $\mathcal{P}_{N}$.

Notation 3. $A^{S}$ represents the attractor obtained from the "switched" solution using the PS scheme, and $A^{*}$ is the attractor of the "averaged" solution when $p=p^{*}$.

If the PS scheme in (5) is applied to any system represented by the initial value problem in Equation (2), like the Chen model, it is possible to approximate any desired attractor of the system by simply switching the control parameter. For example, to approximate a stable orbit of a dynamical system, the set $\mathcal{P}_{N}$ is chosen from the chaotic window of the system, while $p^{*}$ corresponds to a periodic window. Hence, between the chaotic windows, there exists at least one periodic window. It is important to note that the PS scheme does not change the original properties of attractor $A^{S}$.

Remark 1. The uniqueness of having different $p$ values gives the possibility of getting different solutions.

Remark 2. In the practical sense, it is very much feasible to approximate an attractor $A^{*}$ from within a set $\mathcal{P}_{N}$, which does not contain $p^{*}$, i.e., $p^{*} \notin \mathcal{P}_{N}$, but $p^{*}$ is within the real open interval $\left(p_{1}, p_{N}\right)$ because $p^{*}$ is a convex combination of ordered $p_{k}$, where $k=1,2, \ldots, N$. 
Remark 3. The same attractor $A^{S}$ can be obtained with different choices of $\mathcal{P}_{N}$ and $\mathcal{M}_{N}$.

A four-step methodology for the synthesis of attractors by parameter switching is adopted as follows:

Step 1: Select the appropriate switched parameters for the switching scheme and their associated weights.

Step 2: Execute the parameter-switching scheme using the model in Equation (2) to generate the synthesized attractor $A^{S}$ of the "switched" solution.

Step 3: Replace $p$ in model (2) with $p^{*}$, and run to generate attractor $A^{*}$ of the "averaged" solution.

Step 4: Show that the attractor $A^{S}$ of the "switched" solution matches $A^{*}$ of the "averaged" solution.

Remark 4. Without loss of generality, the initial condition $x_{0}$ for the "switched" solution and $y_{0}$ for the "averaged" solution are the same $\left(x_{0}=y_{0}\right)$, in order to reduce the transient steps.

\subsection{Synchronization of Two Chen Oscillators}

Hamiltonian systems with the observer-based approach are employed in this section to synchronize two chaotic Chen oscillators in Equation (1), in a master-slave configuration. The slave unit served as the state observer, which approximates the master unit. A detailed description of the Hamiltonian systems with the observer-based approach is contained in [56]. The following is the mathematical representation of the master and slave units in the Hamiltonian system:

Let $\frac{d x}{d t}=f(x)$ be a dynamical system, in which $x \in \boldsymbol{R}^{n}$ represents the state variable and $f: \boldsymbol{R}^{n} \rightarrow \boldsymbol{R}^{n}$ is the nonlinear function. The Hamiltonian forms can be described as:

$$
\frac{d x}{d t}=\mathcal{J}(x) \frac{\partial H}{\partial x}+\mathcal{S}(x) \frac{\partial H}{\partial x}+\mathcal{F}(x), \quad x \in \boldsymbol{R}^{n}
$$

whereby $H(x)=\frac{1}{2} x^{T} \mathcal{M} x$ is the energy function greater than zero belonging to $\boldsymbol{R}^{n}$, in which $\mathcal{M}$ is a definite matrix greater than zero, constant and symmetric. Therefore, $\frac{\partial H}{\partial x}=\mathcal{M} x$. Furthermore, $\frac{\partial H}{\partial x}$ is the gradient vector derived from $H(x)$. Matrix $\mathcal{J}(x)$ fulfills $\mathcal{J}(x)+\mathcal{J}^{T}(x)=0$, while $\mathcal{S}(x)$ satisfies $\mathcal{S}(x)=\mathcal{S}^{T}(x)$ for all $x \in \boldsymbol{R}^{n}$. The vector field $\mathcal{J}(x) \frac{\partial H}{\partial x}$ is the conservative part of the system. $\mathcal{S}(x)$ represents the nonconservative part. $\mathcal{F}(x)$ is the destabilizing vector.

Therefore, the master system for the case of the observer-based approach, which is a category of the generalized Hamiltonian forms with a single output $y(t)$, is given by:

$$
\left\{\begin{array}{l}
\frac{d x}{d t}=\mathcal{J}(y) \frac{\partial H}{\partial x}+\mathcal{S}(y) \frac{\partial H}{\partial x}+\mathcal{F}(y), \quad x \in \boldsymbol{R}^{n} \\
y=\mathcal{C} \frac{\partial H}{\partial x}, \quad y \in \boldsymbol{R}^{m}
\end{array}\right.
$$

whereby $\mathcal{S}$ represents a matrix, constant and symmetric, and $\mathcal{C}$ is a constant matrix.

The nonlinear slave state observer is constructed from Equation (7) as shown in the next equation:

$$
\left\{\begin{array}{l}
\frac{d \xi}{d t}=\mathcal{J}(y) \frac{\partial H}{\partial \xi}+\mathcal{S}(y) \frac{\partial H}{\partial \xi}+\mathcal{F}(y)+\mathcal{K} e_{y}, \quad \xi \in \boldsymbol{R}^{n} \\
\eta=\mathcal{C} \frac{\partial H}{\partial \zeta}, \quad \eta \in \boldsymbol{R}^{m}
\end{array}\right.
$$

whereby $\xi$ represents the estimate of vector $x, \eta$ is the estimated output, $\mathcal{K}$ represents the observer's gain, while $e_{y}=y-\eta$ stands for the estimated error of the output. The estimated error is calculated as $e=x-\xi$.

Based on the foregoing brief analysis, the master, which is the driving system, and the slave, which is the response system, both of the Chen oscillator (1), are given in Equations (10) and (11), respectively: 


$$
\begin{gathered}
{\left[\begin{array}{l}
\frac{d x}{d t} \\
\frac{d y}{d t} \\
\frac{d z}{d t}
\end{array}\right]=\left[\begin{array}{ccc}
0 & a-\frac{c}{2} & 0 \\
-a+\frac{c}{2} & 0 & -x \\
0 & x & 0
\end{array}\right] \frac{\partial H}{\partial x}+\left[\begin{array}{ccc}
-a & \frac{c}{2} & 0 \\
\frac{c}{2} & c & 0 \\
0 & 0 & -b
\end{array}\right] \frac{\partial H}{\partial x}} \\
{\left[\begin{array}{c}
\frac{d \tilde{\xi}_{1}}{d t} \\
\frac{d \tilde{\xi}_{2}}{d t} \\
\frac{d \tilde{\xi}_{3}}{d t}
\end{array}\right]=\left[\begin{array}{ccc}
0 & a-\frac{c}{2} & 0 \\
-a+\frac{c}{2} & 0 & -x \\
0 & x & 0
\end{array}\right] \frac{\partial H}{\partial x}+\left[\begin{array}{ccc}
-a & \frac{c}{2} & 0 \\
\frac{c}{2} & c & 0 \\
0 & 0 & -b
\end{array}\right] \frac{\partial H}{\partial x}+\left[\begin{array}{l}
k_{1} \\
k_{2} \\
k_{3}
\end{array}\right] e_{y}}
\end{gathered}
$$

The synchronization is said to be successful based on the next definition, in conjunction with the following theorems.

Definition 1. Using the Hamiltonian systems, the synchronization of the master and slave systems occurs when:

$$
\lim _{t \rightarrow \infty}\|x(t)-\xi(t)\|=0
$$

notwithstanding the values of the initial conditions $x(0)$ and $\xi(0)$ [57].

Theorem 1. The global, exponential, and asymptotic estimation of state $x$ of the master system $(10)$ by the state $\xi$ of the slave system $(11)$ is achievable if both matrices $(\mathcal{C}, \mathcal{S})$ are observable [57].

Theorem 2. The global, exponential, and asymptotic estimation of state $x$ of the master system (10) by the state $\xi$ of the slave system (11) is achievable if there is a constant matrix $\mathcal{K}$, in which the symmetric matrix:

$$
[W-K C]+[W-K T]^{T}=[S-K C]+[S-K C]^{T}=2\left[S-\frac{1}{2}\left(K C+C^{T} K^{T}\right)\right]
$$

is negative definite [57].

\section{VHDL Implementation and System Co-Simulation}

This section contains the implementation results of the parameter-switching scheme and master-slave synchronization. They were co-simulated in both MATLAB/Simulink and Active-HDL. The word size for the VHDL coding was 24 bits: the integer part used nine bits, and the fractional part occupied fourteen bits and one bit for the sign.

\subsection{Parameter Switching Implementation}

To numerically implement the parameter-switching scheme, it was required to choose a numerical technique with a fixed step-value $h$ to integrate the underlying initial value problem. The applied numerical method was the Runge-Kutta method (fourth order) where $h=0.001$. To approximate with the PS scheme the solution corresponding to $p^{*}$, it was therefore important to find a set $\mathcal{P}$ and the corresponding set $\mathcal{M}$, such that Equation (6) gives the searched value $p^{*}$. For this work, $N=6$; hence, $\mathcal{P}_{N}=\left\{p_{1}, p_{2}, p_{3}, p_{4}, p_{5}, p_{6}\right\}, \mathcal{M}_{N}=$ $\left\{m_{1}, m_{2}, m_{3}, m_{4}, m_{5}, m_{6}\right\}$, and $\mathcal{A}_{N}=\left\{A^{p_{1}}, A^{p_{2}}, A^{p_{3}}, A^{p_{4}}, A^{p_{5}}, A^{p_{6}}\right\}$. Our $\mathcal{P}_{N}=\{24.75,25.80$, $27.08,27.38,26.38,25.10\}$, selected from the chaotic window in the bifurcation diagram in Figure 2, as parameter $c$ was varied. $\mathcal{M}_{N}=\{3,2,4,1,1,1\}$ was chosen such that Equation (6) was verified, obtaining the "averaged" parameter $p^{*}=26.0858$, which corresponds to the stable window at $c=p^{*}=26.0858$ in Figure 2 . 


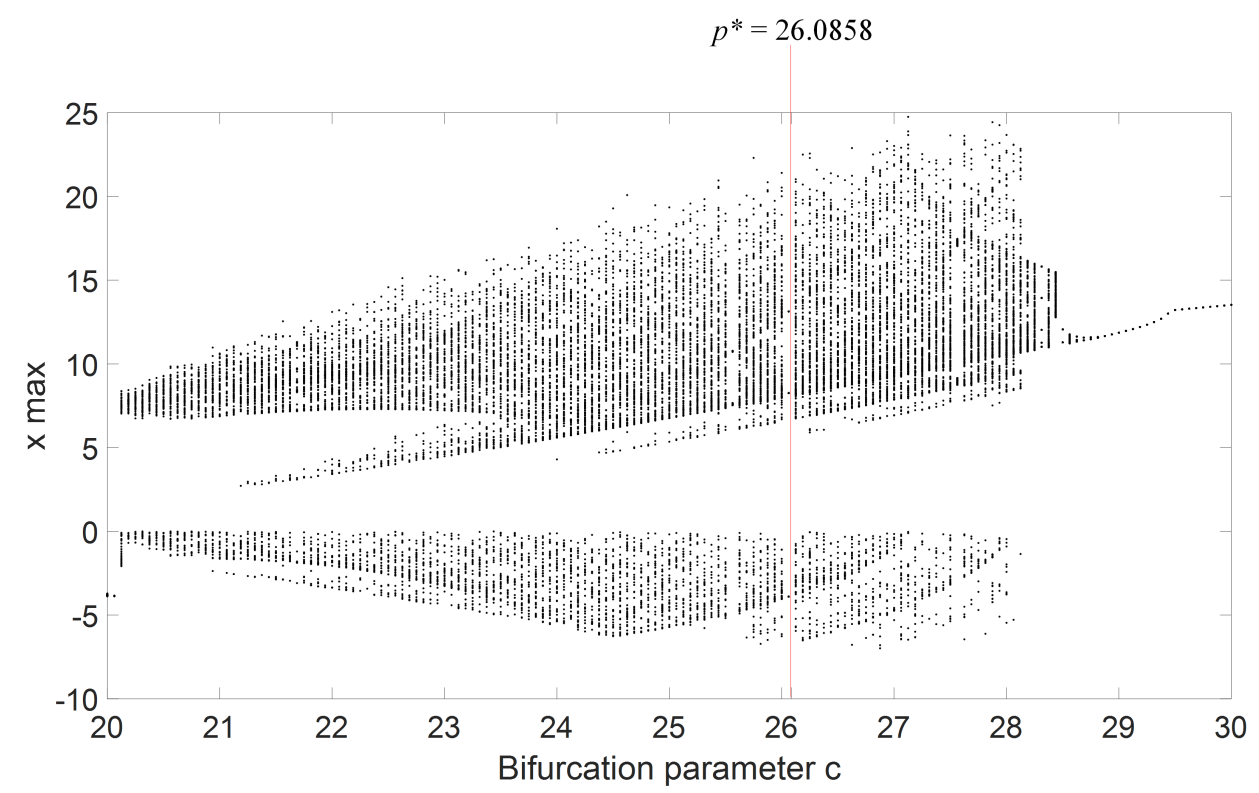

Figure 2. Bifurcation diagram of the Chen oscillator (1) showing the chaotic and stable regions as parameter $c$ is varied. The "averaged" parameter $p^{*}=26.0858$ is selected from the stable window.

The corresponding PS scheme is thus:

$$
\left[m_{1} p_{1}, m_{2} p_{2}, m_{3} p_{3}, m_{4} p_{4}, m_{5} p_{5}, m_{6} p_{6}\right]=\left[3 p_{1}, 2 p_{2}, 4 p_{3}, 1 p_{4}, 1 p_{5}, 1 p_{6}\right]
$$

Several tools can be employed to numerically verify the approximation of the "averaged" attractor $A^{*}$ by the synthesized attractor $A^{S}$. In this investigation, three-dimensional phase plots (after neglecting enough transient period) and time series were used.

For example, to obtain the stable cycle corresponding to the "averaged" parameter $p^{*}$ with our $\mathcal{P}_{N}$ and $\mathcal{M}_{N}$ and using the PS scheme in (14), Equation (6) was evaluated to obtain $p^{*}=26.0858$. The PS scheme in (14) was applied to the IVP in (2) to obtain a synthesized attractor $A^{S}$, which is the numerical approximation of the "averaged" attractor $A^{*}$ from $p^{*}$.

Figure 3a shows the co-simulation diagram of the implementation of the Chen model in Active-HDL with MATLAB/Simulink. Embedded in the SINGLE_P1_CHEN_RK4 block is the numerical solution of the Chen model by fourth-order Runge-Kutta method, while $X, Y$, and $Z$ are the outputs from the state variables. Figure $3 b$ is the block diagram of the VHDL implementation of the PS scheme in Active-HDL. Processes SWITCHER and RK4 implemented the PS scheme with the fourth-order Runge-Kutta method, while $x \_0$, $y \_0$, and $z \_0$ are the outputs.

In Figure 4 are the underlying chaotic attractors $A^{p_{1}}, A^{p_{2}}, A^{p_{3}}, A^{p_{4}}, A^{p_{5}}$, and $A^{p_{6}}$ in $3 \mathrm{D}$ corresponding to parameters $p_{1}=24.75, p_{2}=25.80, p_{3}=27.08, p_{4}=27.38, p_{5}=26.38$, and $p_{6}=25.10$, respectively, obtained from MATLAB and Active-HDL co-simulation of the Chen model using the same parameters and initial condition.

Furthermore, Figures 5 and 6 present the results of the approximation of stable cycles of the Chen oscillator by the parameter-switching algorithm. In Figure 5 are the phase portraits in 3D, showing the "switched" solution $A^{S}$ (red), "averaged" solution $A^{*}$ (blue), and overplots of $A^{S}$ and $A^{*}$, at $p=p^{*}=26.0858$ by the parameter-switching scheme $\left[m_{1} p_{1}, m_{2} p_{2}, m_{3} p_{3}, m_{4} p_{4}, m_{5} p_{5}, m_{6} p_{6}\right]$, where $m_{1}=3, m_{2}=2, m_{3}=4, m_{4}=1, m_{5}=1$, $m_{6}=1$ and $p_{1}=24.75, p_{2}=25.80, p_{3}=27.08, p_{4}=27.38, p_{5}=26.38, p_{6}=25.10$. Figure 6 is the system time series of the three states $x, y$, and $z$ of the Chen system. The time series in MATLAB were plotted by superimposing the "switched" solution over the "averaged" solution to show the matching. The obtained attractors $A^{S}$ and $A^{*}$ were indeed stable cycles. 


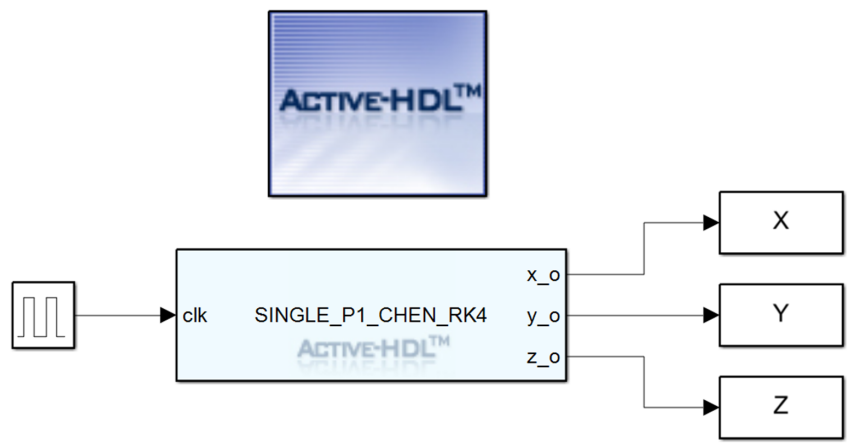

(a)

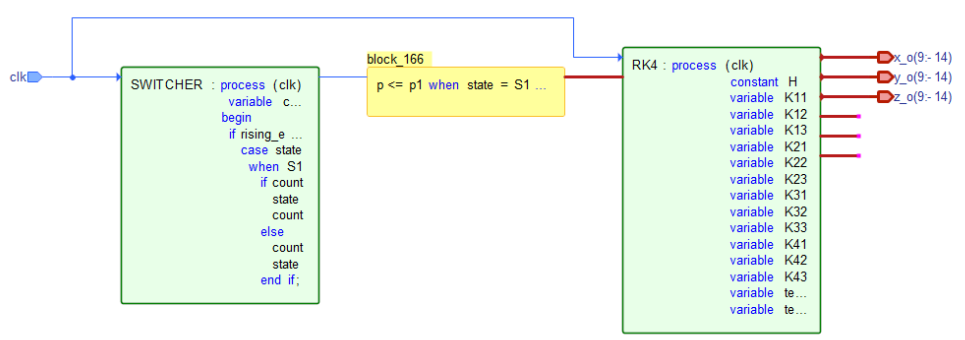

(b)

Figure 3. Chen oscillator and parameter-switching scheme using the 4th-order Runge-Kutta method. (a) Co-simulation in MATLAB/Simulink with Active-HDL. (b) Active-HDL block diagram of the VHDL implementation.
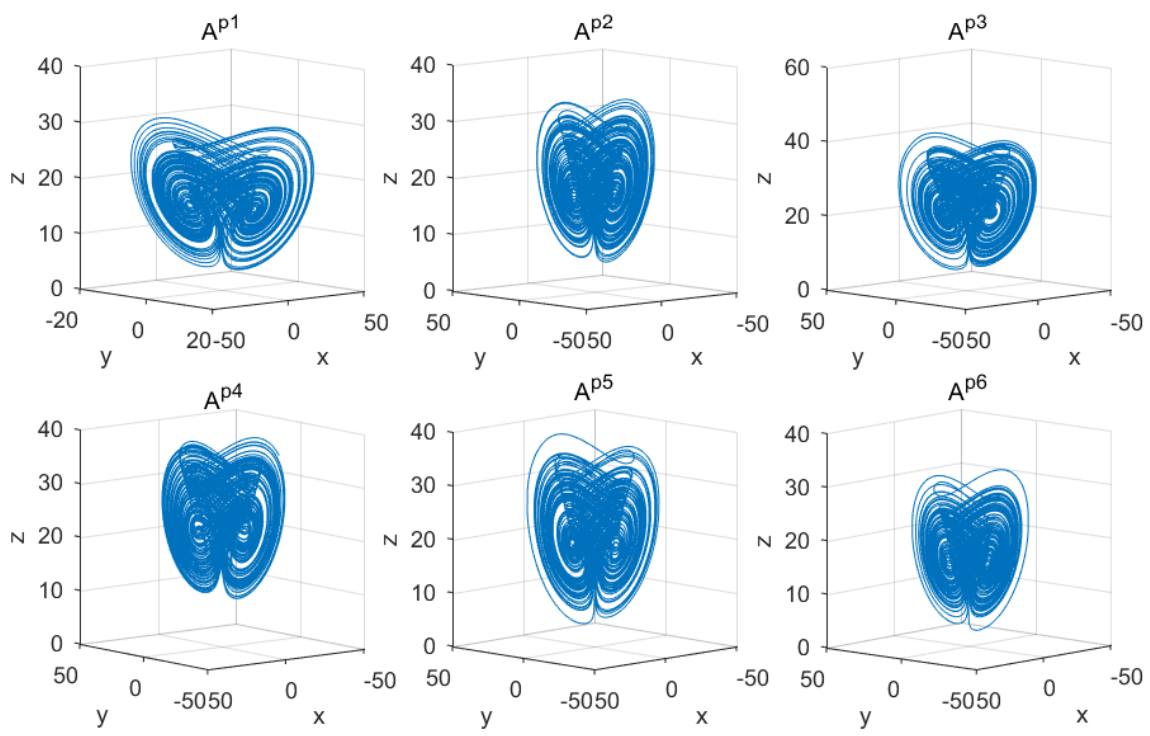

Figure 4. Chaotic phase portraits of the Chen system (1) for the set $\mathcal{A}_{N}$ corresponding to $\mathcal{P}_{N}$. 


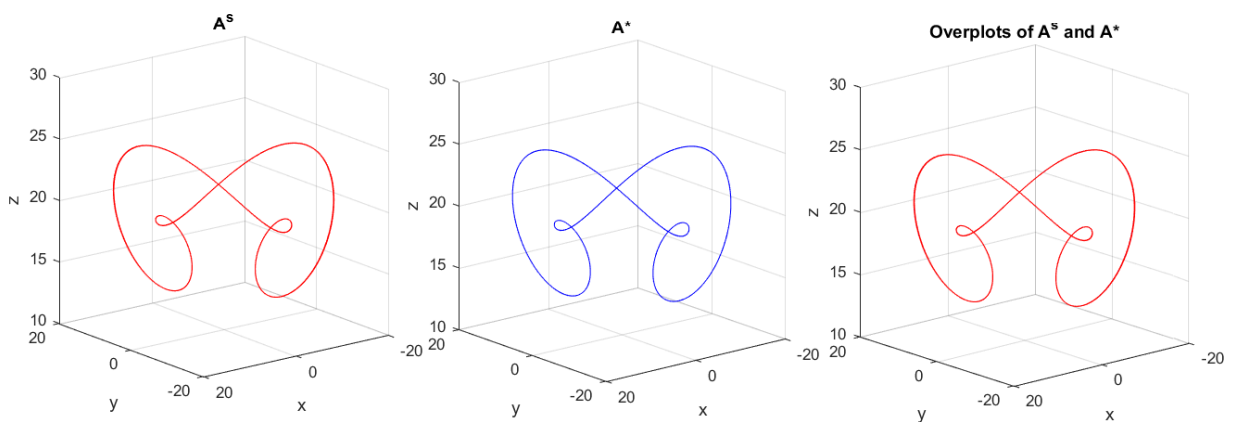

Figure 5. Co-simulation results of the approximation of the Chen system's stable cycle, illustrating the "switched" solution $A^{S}$ (red), "averaged" solution $A^{*}$ (blue), and overplots of $A^{S}$ and $A^{*}$.
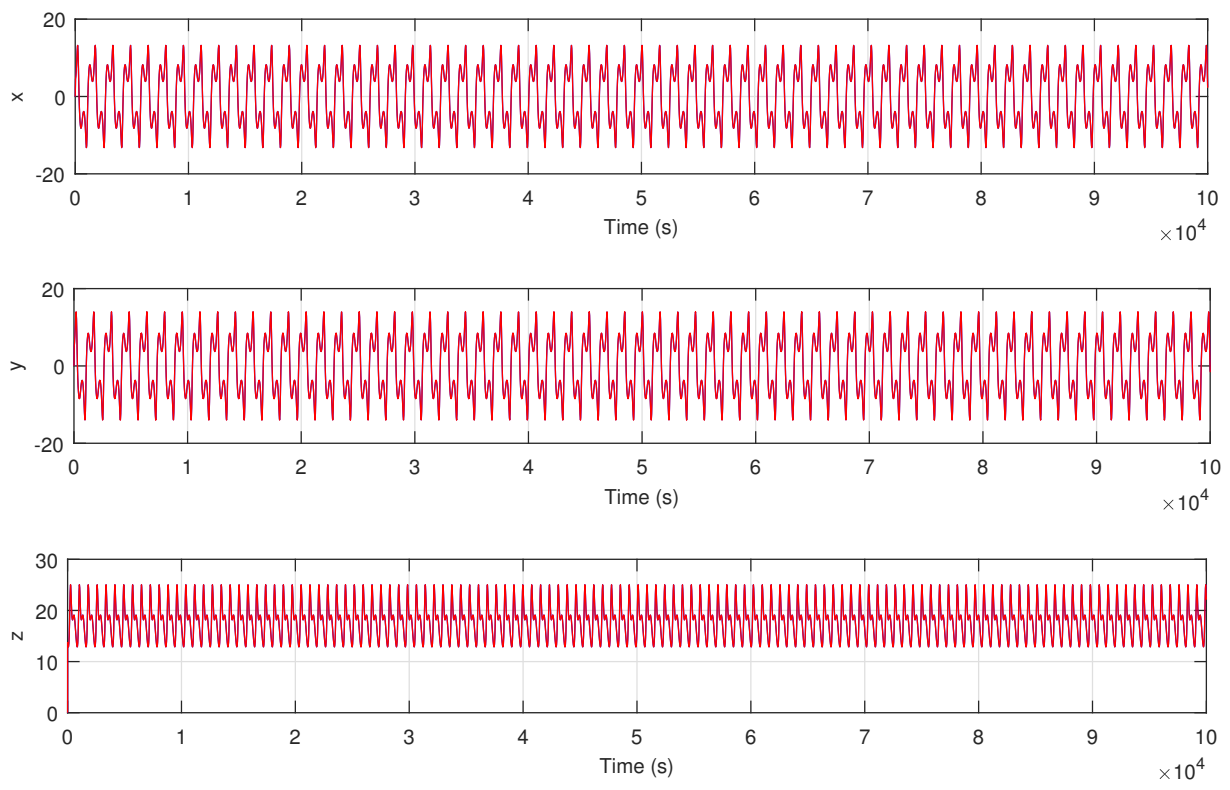

(a)

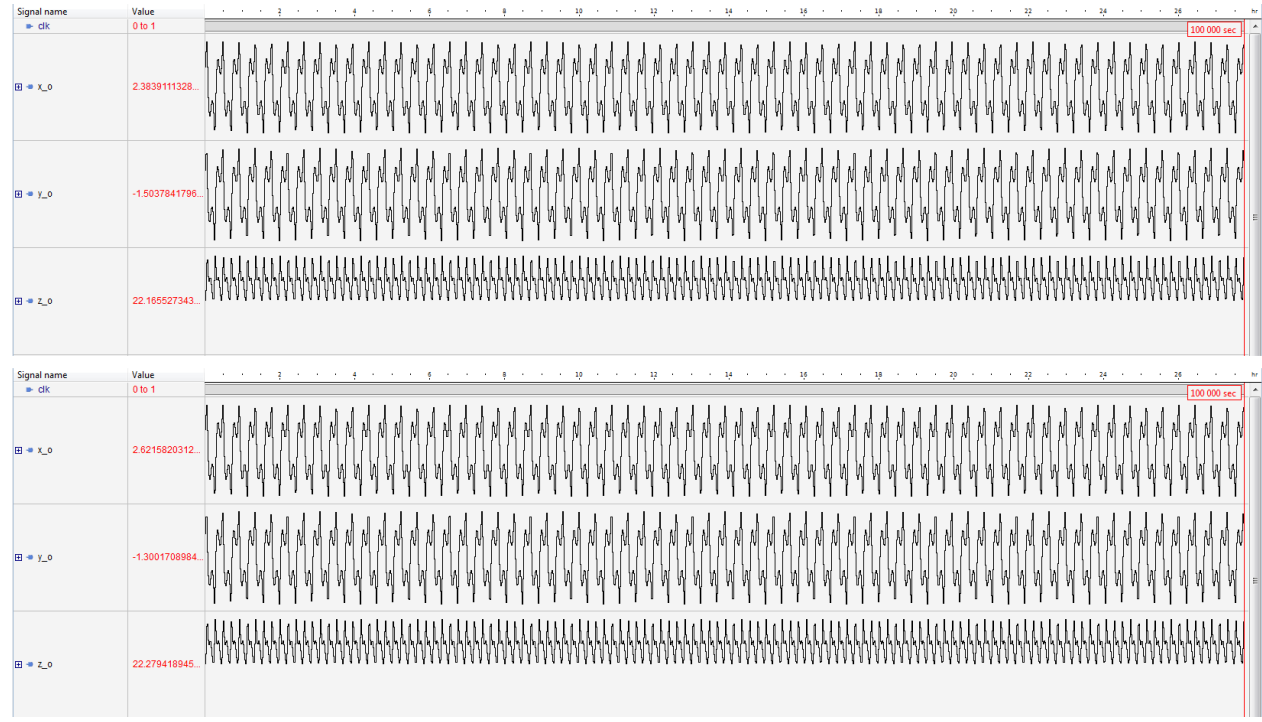

(b)

Figure 6. Time series of the three states $x, y$, and $z$. (a) MATLAB overplots: "switched" solution $A^{S}$ (red) and "averaged" solution $A^{*}$ (blue). (b) Active-HDL: "switched" solution $A^{S}$ (upper) and "averaged" solution $A^{*}$ (lower). 
Looking closely at the considered PS scheme, it is noted that the stable attractors $A^{S}$ were synthesized from the combination of six underlying unstable attractors $A^{p_{1}}, A^{p_{2}}, A^{p_{3}}$, $A^{p_{4}}, A^{p_{5}}$, and $A^{p_{6}}$ in Figure 4 . This can be expressed in the form:

$$
\left[\begin{array}{c}
A^{p_{1}} \\
\text { Chaos }
\end{array}\right]+\left[\begin{array}{c}
A^{p_{2}} \\
\text { Chaos }
\end{array}\right]+\left[\begin{array}{c}
A^{p_{3}} \\
\text { Chaos }
\end{array}\right]+\left[\begin{array}{c}
A^{p_{4}} \\
\text { Chaos }
\end{array}\right]+\left[\begin{array}{c}
A^{p_{5}} \\
\text { Chaos }
\end{array}\right]+\left[\begin{array}{c}
A^{p_{6}} \\
\text { Chaos }
\end{array}\right]=\left[\begin{array}{c}
A^{S} \\
\text { Order }
\end{array}\right]
$$

The expression in (15) is akin to the generalized Parrondo's paradox [55,58,59]. In this situation, our PS scheme served as the chaos control mechanism in the Chen oscillator.

\subsection{Master-Slave Synchronization}

In this work, the system parameter values for the synchronization of the Chen oscillators were $a=35, b=3$. For parameter $c$, all six values in the set $\mathcal{P}_{N}$ were separately used. The chosen gains of the observer were $k_{1}=2, k_{2}=100$, and $k_{3}=0$. The synchronization errors were computed and plotted as $e_{1}=x_{1}-\xi_{1}, e_{2}=x_{2}-\xi_{2}, e_{3}=x_{3}-\xi_{3}$, in which $x_{i}$ is the master system and $\xi_{i}$ is the slave system. The phase error between $x_{i}$ and $\xi_{i}$ is plotted as well. The total number of samples was $10 \times 10^{4}$. Figure 7 a shows the co-simulation diagram of the synchronization in MATLAB/Simulink, whereby XM1, YM1, and ZM1 are the outputs of the master system, $X S 1, Y S 1$, and $Z S 1$ are the outputs of the slave system, while $E 1, E 2$, and $E 3$ are the synchronization errors. The block SYNC_TOP_SINGLE_P contains the function that performs the synchronization of the master and slave systems. Figure $7 \mathrm{~b}$ represents the Active-HDL block diagram in the VHDL implementation. The entities Master and Slave represent the master and slave systems, respectively, while entity Sync handles the synchronization process. For the master system, the outputs are $x m, y m$, and $z m$; for the slave system, the outputs are $x_{s}, y s$, and $z s$; while the synchronization errors are $e 1, e 2$, and $e 3$.

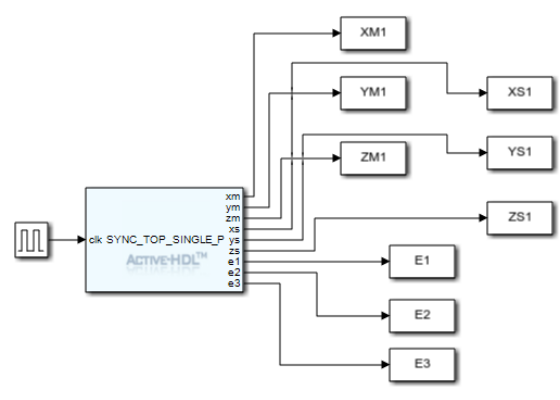

(a)

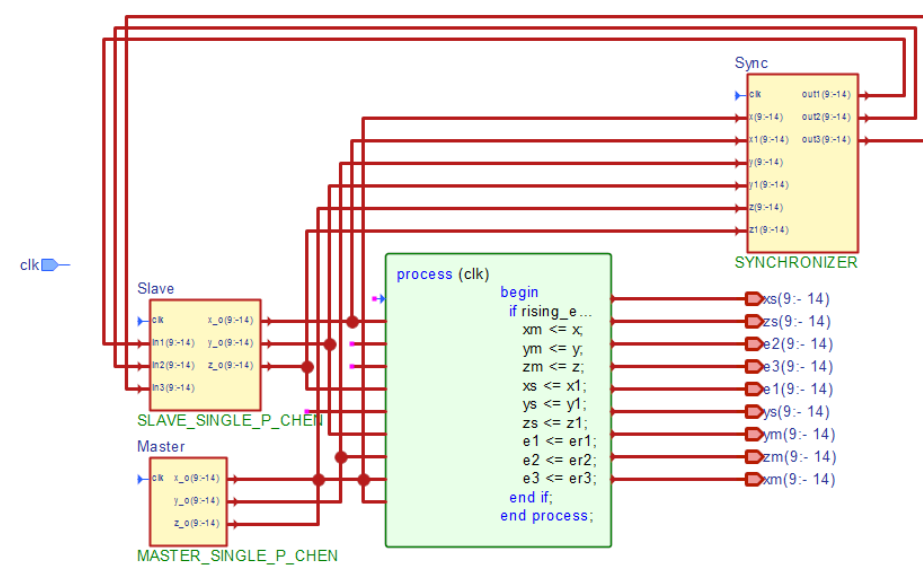

(b)

Figure 7. Hamiltonian synchronization of master and slave Chen systems. (a) Co-simulation in MATLAB/Simulink with Active-HDL. (b) Active-HDL block diagram of the VHDL implementation.

Two cases of synchronization, co-simulated in MATLAB and Active-HDL, are presented in this section. In the first case, the synchronization used different initial conditions as follows: $x_{0}=(4.075,4.425,13.73)$ for the master system and $\xi_{0}=(3,3.5,10.5)$ for the slave system. The second case used the same initial condition, $x_{0}=(4.075,4.425,13.73)$, for the master system, as well as the slave system. Initial conditions of the systems were randomly selected from the basins of attraction, out of the many possible values. The basins of attraction of the Chen system (1) can be visualized in Figure 8. It was computed using the 
Newton-Raphson method [60,61]. To plot the basins, the chosen upper and lower bounds within the state space were $x=[-25,25], y=[-25,25]$, and $z=[-30,50]$. The points in red color converge to the equilibrium point $E P_{0}$ represented by the black diamond shape, while those in green and blue converge to the equilibrium points $E P_{1}$ and $E P_{2}$ indicated by the two black circles, respectively. The eigenvalues at the three equilibria had at least one positive value, hence the possibility of strange attractors.

Both the master and slave chaotic oscillators were sensitive to the respective initial values. This was confirmed by computing the Lyapunov exponents, which for each initial condition had a positive value. In both cases, the master and slave phase diagrams, the phase errors, and the synchronization errors were analyzed. Perfect synchronization was achieved, considering the synchronization and phase error plots, for all the values of parameter $c$ starting with $c=p_{1}$, in which the error signals $e_{1}, e_{2}$ and $e_{3}$ became zero (the same results were obtained for $p_{2}-p_{6}$ ). Therefore, suffice it to say that the ratio between the master state $x_{i}$ and slave state $\xi_{i}$ was one when the synchronization was achieved.

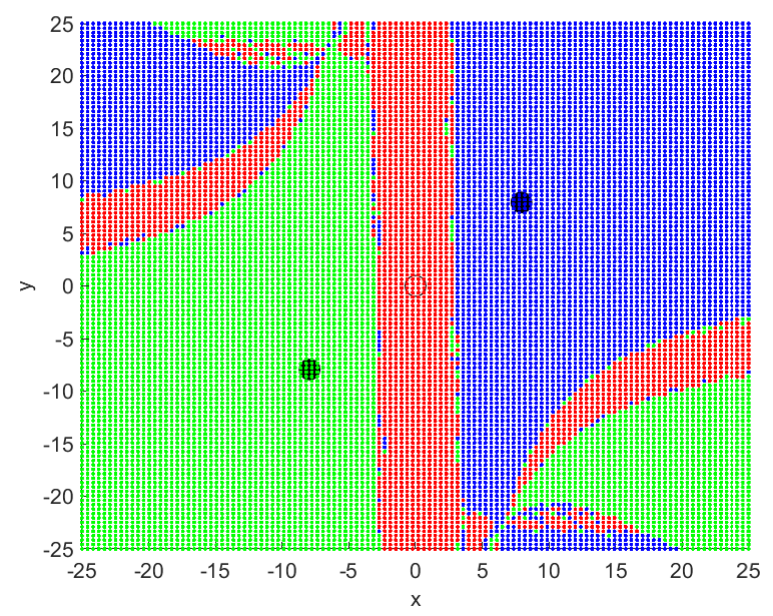

Figure 8. Basins of attraction of the Chen system (1) on $x-y$ plane at $a=35, b=3$, and $c=24.75$; equilibrium point $E P_{0}$ has the red regions as its basin; equilibrium point $E P_{1}$ has green regions as its basin; while equilibrium point $E P_{2}$ has blue regions as its basin.

\section{Application in Image Transmission}

In this section, the synchronized Chen oscillators in the previous section are applied to develop a system for secure communication, to transmit grayscale and RGB images of size $640 \times 480$ pixels $(307,200$ words) and $320 \times 240$ pixels (230,400 words), respectively. Furthermore, the implementation of the image transmission system was co-simulated in both MATLAB/Simulink and Active-HDL. The main attraction here was the application of the parameter-switching technique to decrypt a transmitted (encrypted) image. In the communication concept, the master system is the transmitter subsystem, while the slave system is the receiver subsystem. The co-simulation diagram of the image transmission system in Simulink with Active-HDL and the Active-HDL block diagram are shown in Figure 9, respectively. In Figure 9a, img_origin, encrypted_column, and $i m g \_r x \_c o l u m n$ from the output ports generate the original image, the encrypted image, and the decrypted image signals, respectively, in MATLAB, and the function that coordinates the image transmission is embedded in the TRANSMISSION_TOP block. On the other hand, Figure $9 \mathrm{~b}$ contains four entities, namely Master, which is the master system, Slave, the slave system, Sync, the synchronization unit, and TXN_RXN, the unit that handles the actual image transmission. The original, encrypted, and decrypted image signals were received via image_original, encrypted, and image_out output ports. 


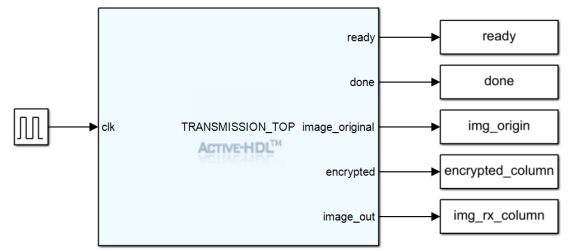

(a)

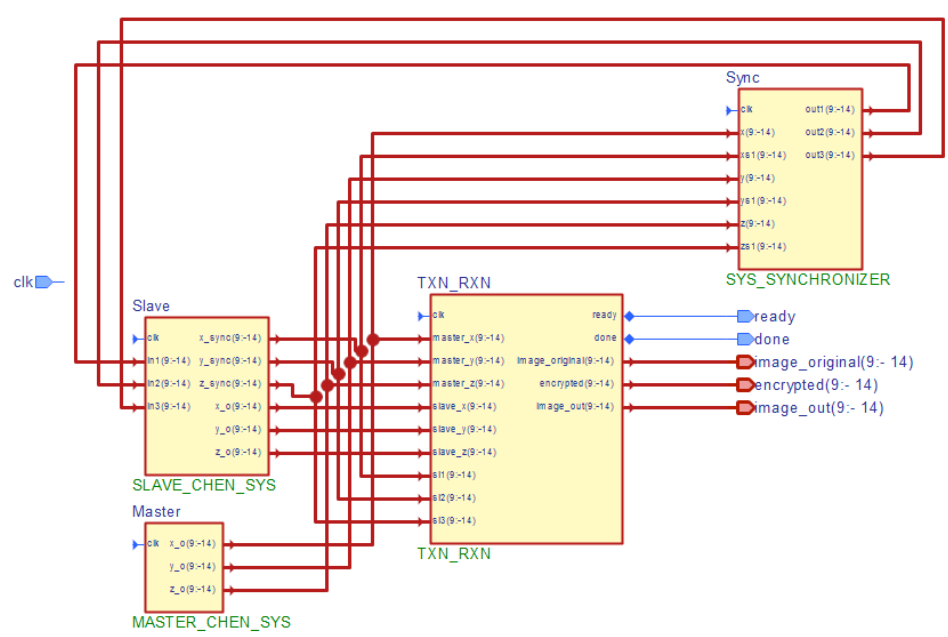

(b)

Figure 9. Image transmission system. (a) Co-simulation diagram in MATLAB/Simulink with Active-HDL. (b) Active-HDL block diagram.

The image transmission system is briefly described in the following threestep methodology:

Step 1: Creating the original RGB and grayscale image data in VHDL.

Step 2: Encrypting the original image with the chaotic state of the master system, e.g., master_x , in the TXN_RXN entity in Figure 8 (the master system is developed with any of the parameters in $\mathcal{P}_{N}$ ).

Step 3: Recovering the image data, e.g., image_out, from the encrypted signal by removing chaos using the corresponding state of the slave system, e.g., slave_ $x$.

In this work, the three states $x, y$, and $z$ were applied as transmission variables to encrypt and decrypt the images. The total simulation time for the RGB image transmission was 234,400 samples and 311,200 samples for grayscale image transmission. It is important to state that the initial conditions and the system parameters were private keys in the encryption and decryption, making the system a symmetric one. The co-simulation results from Active-HDL with Simulink, showing the original, encrypted, and received image data, are shown in Figure 10 for the grayscale image and Figure 11 for the RGB image. In both figures, the original images (in red color) are mixed with chaos to give the encrypted images (in blue color). Moreover, with respect to Figure 9, the original image signals were obtained from the img_origin and image_origin ports, encrypted image signals from the encrypted_column and encrypted ports, and the received image signals via the img_rx_column and image_out ports.

In Figure 12a,b are found the actual original, encrypted, and recovered grayscale and RGB images obtained from our best result, respectively. Statistical analyses were performed in MATLAB to find the correlation between the original and encrypted images, encrypted and received images, and original and received images. The results are presented in Table 1. 
Table 1. Correlation coefficients' analysis of the original, encrypted, and received RGB and grayscale images, using the parameter-switching scheme for image decryption.

\begin{tabular}{clcccc}
\hline \multirow{2}{*}{$\begin{array}{c}\text { Transmission } \\
\text { Variable }\end{array}$} & Correlation & \multicolumn{3}{c}{ RGB Image } & \multirow{2}{*}{$\begin{array}{l}\text { Grayscale } \\
\text { Image }\end{array}$} \\
\cline { 3 - 5 } & & Red & Green & Blue & 0.0352 \\
\multirow{2}{*}{$x$} & Original and Encrypted & 0.0342 & 0.0543 & 0.0315 & 0.0352 \\
& Encrypted and Received & 0.0342 & 0.0543 & 0.0315 & 1 \\
\hline \multirow{2}{*}{$y$} & Original and Received & 1 & 1 & 1 & 0.0362 \\
& Original and Encrypted & 0.0316 & 0.0498 & 0.0296 & 0.0362 \\
& Encrypted and Received & 0.0316 & 0.0498 & 0.0296 & 1 \\
\hline \multirow{2}{*}{$z$} & Original and Received & 1 & 1 & 1 & 0.0672 \\
& Original and Encrypted & 0.0649 & 0.0747 & 0.0711 & 0.0672 \\
& Encrypted and Received & 0.0649 & 0.0747 & 0.0711 & 1 \\
\hline
\end{tabular}
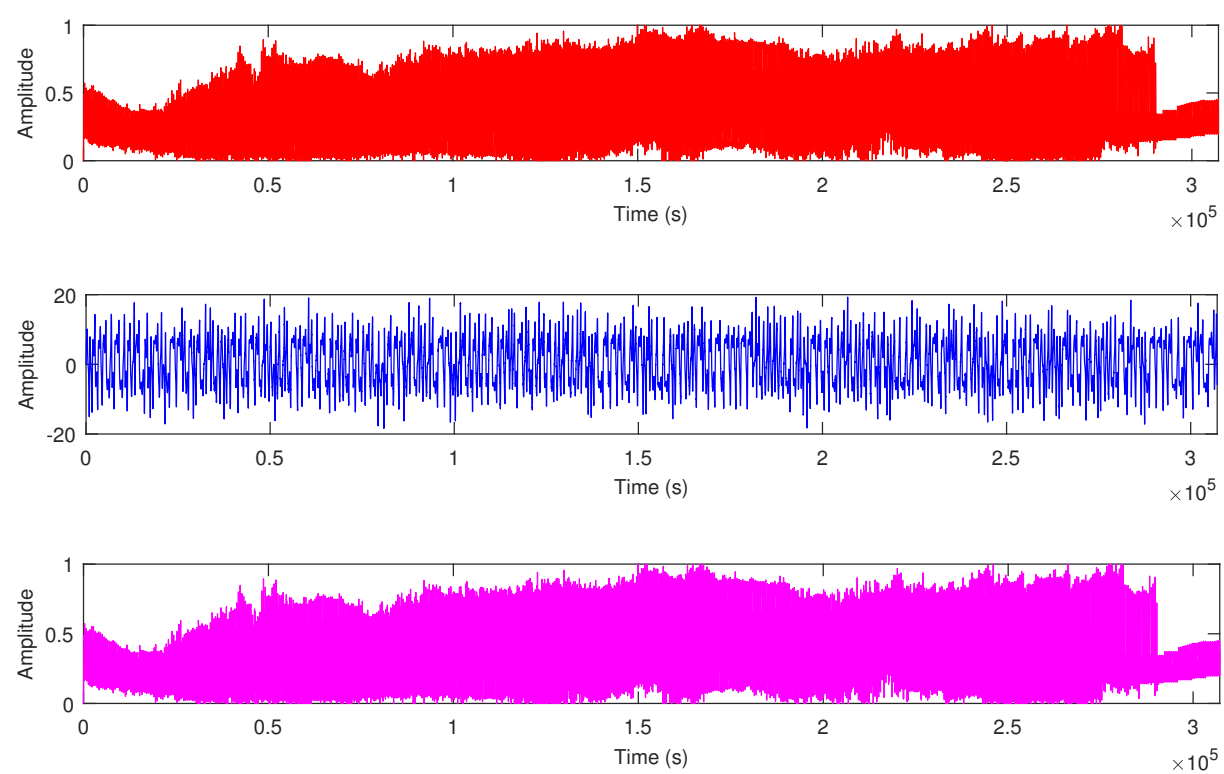

(a)

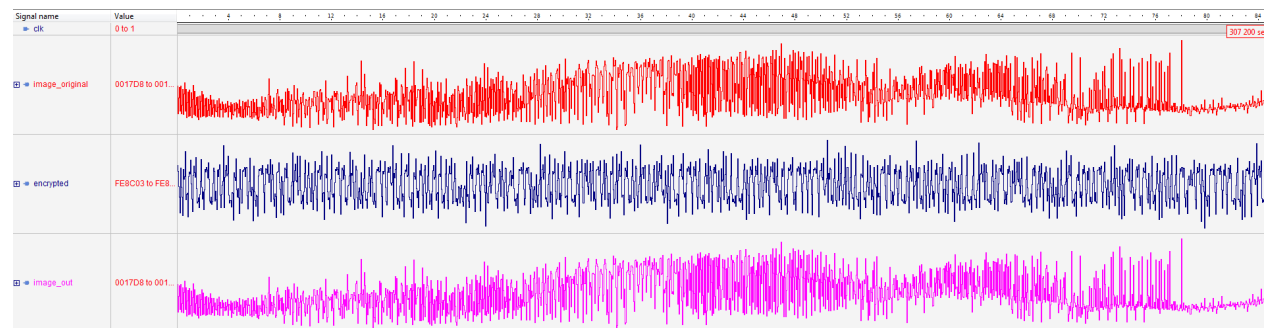

(b)

Figure 10. Waveforms of the $640 \times 480$ grayscale image from transmission variable $x$, showing the original (red), encrypted (blue), and received (magenta) recovered by the parameter-switching technique. (a) Co-simulation diagram in MATLAB/Simulink with Active-HDL. (b) Active-HDL. 

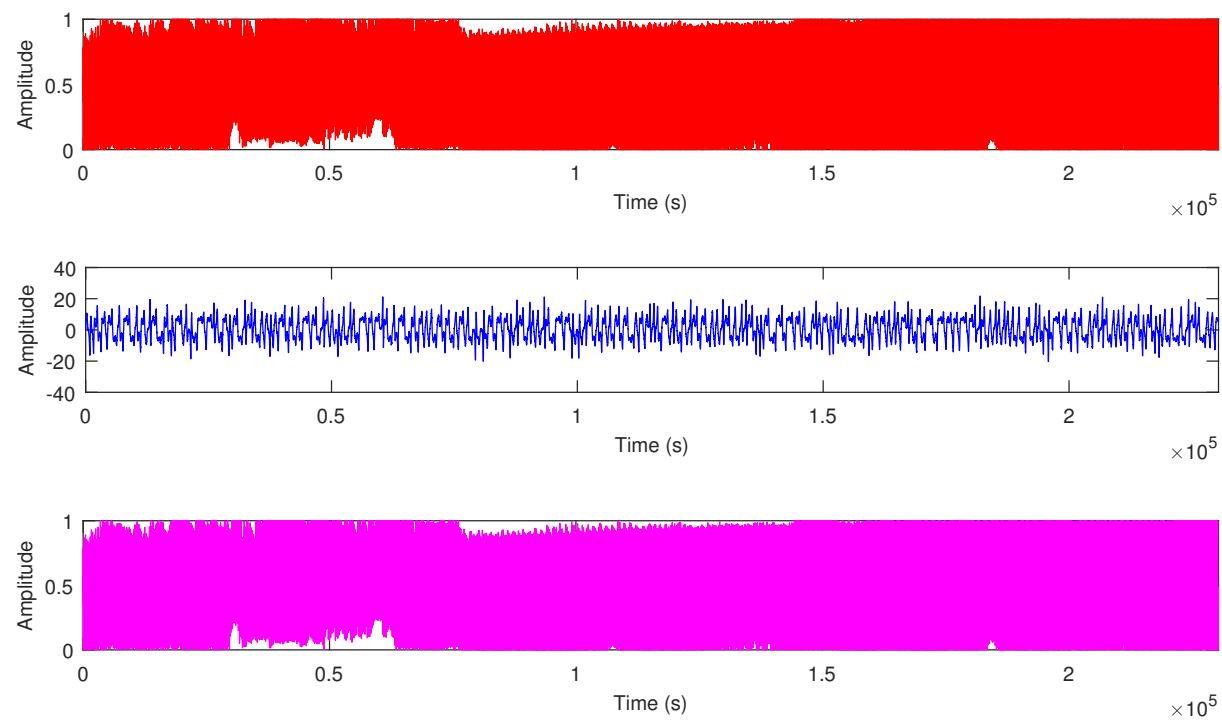

(a)

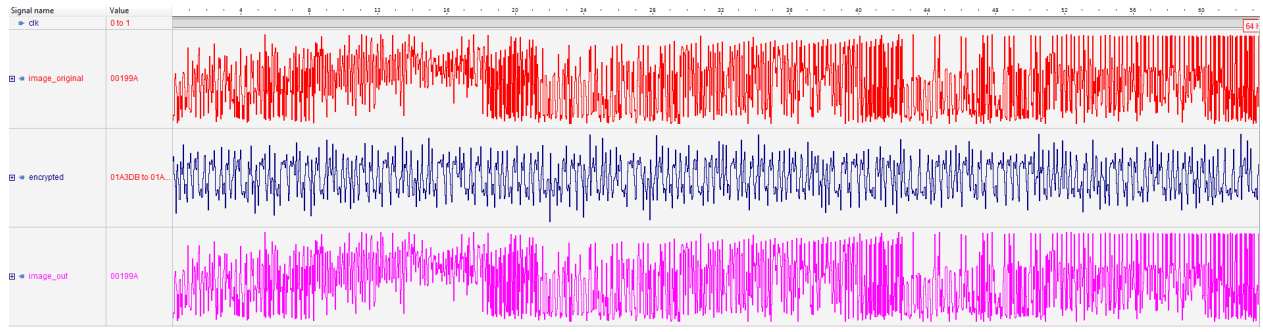

(b)

Figure 11. Waveforms of the $320 \times 240$ RGB image data from transmission variable $y$, showing the original (red), encrypted (blue), and received (magenta) recovered by the parameter-switching technique. (a) Co-simulation diagram in MATLAB/Simulink with Active-HDL. (b) Active-HDL.
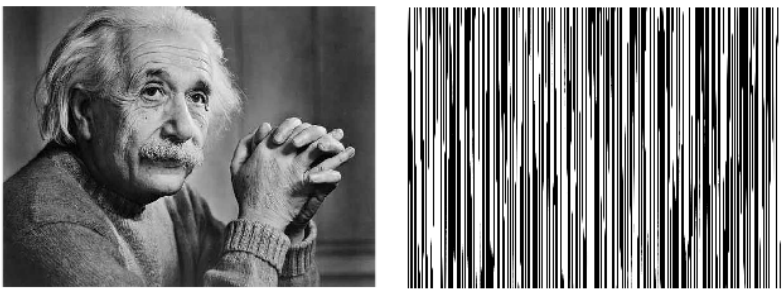

(a)
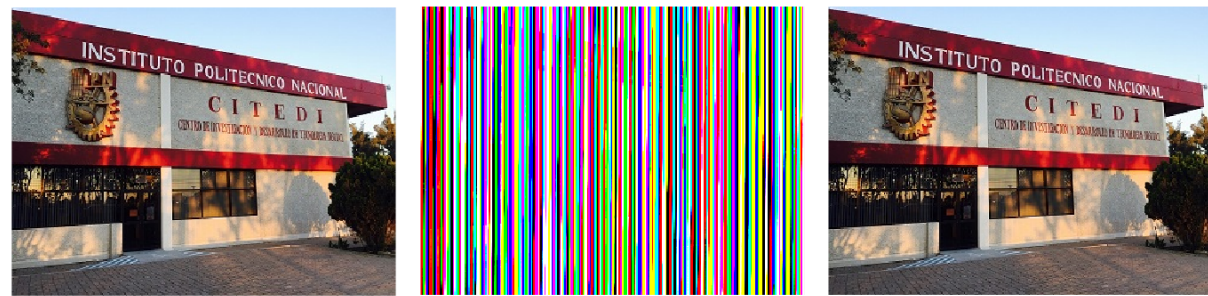

(b)

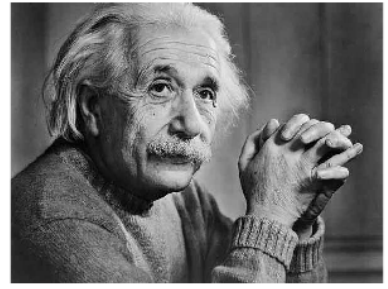




\section{Discussion}

The nonlinearities inherent in the Chen model (1) help give rise to the chaotic attractors in Figure 4. Upon the application of the parameter-switching scheme to evaluate the Chen model, effectively evaluating six different Chen chaotic systems in turn in the process, a stable Chen system was obtained. Suffice it to say that chaos in the underlying six chaotic attractors was controlled to give rise to a stable cycle (see the "switched" solution $A^{S}$ in Figure 5).

The backbone of the image transmission system in Figure 9 was the synchronized master and slave Chen systems. The slave unit Slave had embedded in it a parameter switching unit, which used the scheme in (14) to stabilize chaos in the master system to recover the encrypted image data. In the results presented in Figures 10-12, each pixel of the original grayscale and RGB image data was blended with the chaotic state of the master unit to create the encrypted image data. Specifically, in Figures 10 and 11, it is observed that the encrypted image data became random. The randomness in the encrypted images rendered them meaningless to potential hackers. After the images were reconstructed, the randomness was removed, and it can be seen that the recovered images were exactly the same as the original images. This result was significant because the recovery was achieved by applying the parameter-switching technique.

In the correlation results in Table 1, it is shown that with a correlation of one between the original and received images for both the RGB and grayscale images, the transmitted images were recovered fully without any loss of quality by applying the parameter-switching technique for the decryption. Considering the security of the encrypted images, given by the correlation between the original and encrypted images, our best result was obtained from variable $y$ for the RGB images and $x$ for the grayscale image. As a matter of fact, the correlations between the original and encrypted grayscale and RGB images, as well as between the encrypted and received grayscale and RGB images were the same, meaning that both the original and received images were the same. Moreover, the computed mean-squared error (MSE) was zero; hence, the peak-signal-to-noise ratio (PSNR) tended to infinity. This revelation was attributed to the fact that the chaos-based transmission system was resistant to noise and distortion [62].

All the work carried out in this investigation, namely the implementation of the Chen oscillator, the synthesis of attractors by the parameter-switching scheme, the synchronization of Chen oscillators, and image transmission, was realized on an FPGA device. As stated earlier, the HDL used was VHDL, and the size of the digital block was twenty-four bits: the integer segment used nine bits; the fraction occupied fourteen bits and one bit for the sign. The FPGA emulations were performed on the Xilinx Artix-7 AC701 board Device Number XC7A200TFBG676-2. The FPGA resources consumed by the activities listed above are presented in Table 2. As we can see, each of the activities required only less than one percent of the total registers on the FPGA board for the implementation. The same amount of FPGA resources was utilized to implement the Chen chaotic oscillator and the parameter-switching scheme to approximate stable cycles of the Chen oscillator.

Table 2. Utilization of FPGA resources in the Xilinx Artix-7 AC701 board to implement the: (1) Chen chaotic oscillator, (2) parameter-switching scheme applied to approximate stable cycles of the Chen oscillator, and (3) the Hamiltonian form synchronization of Chen oscillators.

\begin{tabular}{lrcccccc}
\hline & \multicolumn{2}{c}{ Chen Oscillator } & Parameter Switching & \multicolumn{2}{c}{ Synchronization } \\
\hline Resources & Available & Used & $\begin{array}{c}\text { Utilization } \\
\mathbf{( \% )}\end{array}$ & Used & $\begin{array}{c}\text { Utilization } \\
\mathbf{( \% )}\end{array}$ & Used & $\begin{array}{c}\text { Utilization } \\
\mathbf{( \% )}\end{array}$ \\
\hline Slice LUTs & 134,600 & 5615 & 4 & 5472 & 4 & 11,935 & 9 \\
Memory LUTs & 46,200 & 0 & 0 & 0 & 0 & 0 & 0 \\
Registers & 269,200 & 144 & $<1$ & 179 & $<1$ & 479 & $<1$ \\
I/O pins & 400 & 73 & 18 & 73 & 18 & 145 & 36 \\
Block RAMs & $13,140,000$ & 0 & 0 & 0 & 0 & 0 & 0 \\
DSPs & 740 & 44 & 6 & 40 & 5 & 64 & 9 \\
\hline
\end{tabular}


Table 3 exhibits the utilization of FPGA resources for the image transmission, the grayscale consumed more resources than the RGB, in particular with the amount of utilized slice LUTs. The difference in the slice LUTs utilization was attributed to the size of the images, which were 307,200 words for the grayscale and 230,400 words for the RGB image.

Table 3. Utilization of FPGA resources in the Xilinx Artix-7 AC701 board to implement RGB and grayscale image transmissions by Chen oscillators using the parameter-switching scheme for decryption.

\begin{tabular}{lrrccc}
\hline \multirow{2}{*}{ Resources } & \multirow{2}{*}{ Available } & \multicolumn{2}{c}{ RGB Image } & \multicolumn{2}{c}{ Grayscale Image } \\
\cline { 3 - 6 } & & Used & Utilization (\%) & Used & Utilization (\%) \\
\hline Slice LUTs & 134,600 & 73,157 & 54 & 83,810 & 62 \\
Memory LUTs & 46,200 & 0 & 0 & 0 & 0 \\
Registers & 269,200 & 885 & $<1$ & 948 & $<1$ \\
I/O pins & 400 & 75 & 19 & 75 & 19 \\
Block RAMs & $13,140,000$ & 0 & 0 & 0 & 0 \\
DSPs & 740 & 104 & 14 & 104 & 14 \\
\hline
\end{tabular}

The secure communication system realized on the Intel's Artix-7 AC701 board in this investigation was compared with some other implementations [38,63-65], using some of the FPGA resources and other parameters such as the type of board, the numerical method applied, the language of implementation, and the format of the fixed-point number. This is presented in Table 4. The FPGA resource consumption on Artix-7 AC701 performed favorably against the compared works.

Table 4. Comparing this work with other FPGA implementations of secure communication systems.

\begin{tabular}{lcccccc}
\hline Parameter & This Work (RGB) & This Work (Grayscale) & Ref. [38] & Ref. [63] & Ref. [64] & Ref. [65] \\
\hline FPGA & Artix-7 & Artix-7 & ZYNQ & Cyclone IV & Stratix IV & Virtex 5 \\
Slice LUTs & $54 \%$ & $62 \%$ & $43 \%$ & $33 \%$ & $27 \%$ & $24 \%$ \\
Registers & $<1 \%$ & $<1 \%$ & $25 \%$ & $27 \%$ & $<1 \%$ & $5 \%$ \\
I/O pins & $19 \%$ & $19 \%$ & $24 \%$ & N/A & $22 \%$ & $32 \%$ \\
Block RAMs & $0 \%$ & $0 \%$ & N/A & $96 \%$ & $40 \%$ & N/A \\
DSPs & $14 \%$ & $14 \%$ & N/A & $24 \%$ & $7 \%$ & $62 \%$ \\
Algorithm & RK-4 & RK-4 & RK-4 & Euler & Euler & RK-4 \\
Language & VHDL & VHDL & Verilog & Verilog & VHDL & VHDL \\
Number & 24 bit & 24 bit & 32 bit & 32 bit & 19 bit & 32 bit \\
\hline
\end{tabular}

At this juncture, it is important to state that, compared to other systems of communication, like the spread spectrum [66,67], the chaos-based image transmission system implemented in this investigation had the advantage of simplicity. The major subsystems were the master (transmitter) and the slave (receiver), unlike the traditional methods, which have many subsystems. Another merit is that the implemented system employed the chaotic states for both synchronization and encryption, unlike the other communication methods, which use a tool for synchronization and another for encryption. Furthermore, in terms of the number of rounds needed for encryption and decryption, our method used just a single round for each operation, as against several rounds in methods such as the Advanced Encryption Standard (AES) and the Data Encryption Standard (DES) [68,69]. Moreover, the chaos-based transmission system was resistant to noise and distortion. Lastly, our encryption system was less susceptible to security attacks because of the very large key space provided by the parameters and the initial condition.

\section{Conclusions}

The digital implementation of the parameter-switching scheme as the chaos control mechanism was highlighted in this paper. In another situation, parameter switching can function as the chaos anti-control mechanism, whereby two or more underlying stable 
attractors are combined to synthesize a chaotic attractor. The first major contribution of this work was the FPGA realization of the parameter-switching scheme to approximate stable cycles of the Chen oscillator. The second major contribution was the FPGA realization of a secure communication system whereby the parameter-switching scheme was applied as a decryption mechanism to recover chaos-encrypted RGB and grayscale images. In addition, the Chen chaotic model and master-slave synchronization by Hamiltonians and the observer-based approach were also realized on an FPGA device. In each activity, co-simulation results in MATLAB/Simulink and Active-HDL were presented to verify the FPGA realizations. In the case of the image transmission, the results of our experiment confirmed that the method implemented in this work was beneficial to secure communication systems. The bit depth of the grayscale image was eight bits, while the RGB was twenty-four bits, but our method is applicable to images of any bit depth.

Author Contributions: Conceptualization, J.-C.N.-P., V.-A.A., F.-J.P.-P., and E.T.-C.; methodology, J.-C.N.-P., V.-A.A., and E.T.-C.; software, V.-A.A. and Y.S.I.; validation, J.-C.N.-P., Y.S.I., F.-J.P.-P., and E.T.-C.; formal analysis, J.-C.N.-P., V.-A.A., Y.S.I., and E.T.-C.; investigation, J.-C.N.-P., V.-A.A., and F.-J.P.-P.; resources, J.-C.N.-P. and E.T.-C.; writing-original draft preparation, J.-C.N.-P., V.-A.A., and E.T.-C.; writing-review and editing, J.-C.N.-P., V.-A.A., Y.S.I., F.-J.P.-P., and E.T.-C.; visualization, V.A.-A., F.-J.P.-P., and Y.S.I.; supervision, J.-C.N.-P. and E.T.-C.; project administration, J.-C.N.-P.; funding acquisition, J.-C.N.-P. All authors read and agreed to the published version of the manuscript.

Funding: The authors wish to thank the Instituto Politecnico Nacional for its support provided through the project SIP-20210345. In addition, the authors would like to express their gratitude to the COFAA-IPN for its financial support.

Institutional Review Board Statement: Not applicable.

Informed Consent Statement: Not applicable.

Data Availability Statement: Not applicable.

Conflicts of Interest: The authors declare no conflict of interest.

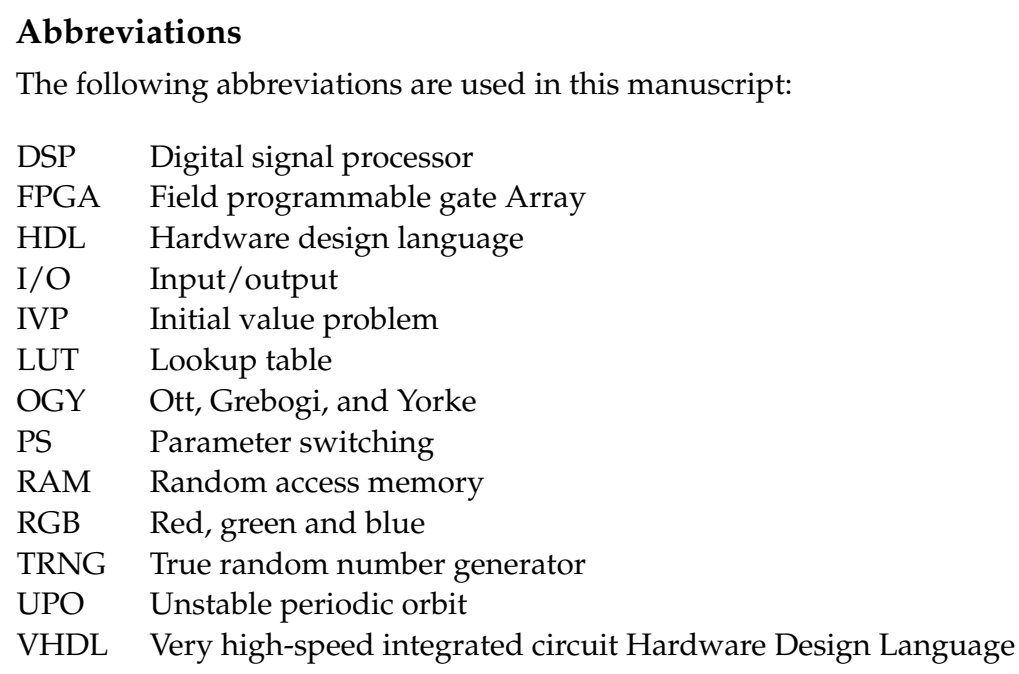

\section{References}

1. Nicolis, G. Chapter 1-The many facets of complexity. In Complexity Science: An Introduction; Peletier, M.A., van Santen, R.A., Steur, E., Eds.; World Scientific Publishing Co.: Singapore, 2019; pp. 3-30. ISBN 978-981323960-9.

2. Hu, M.; Li, F. A new method to solve numeric solution of nonlinear dynamic system. Math. Probl. Eng. 2016, $2016,1485759$. [CrossRef]

3. Li, X.; Liao, S. Clean numerical simulation: A new strategy to obtain reliable solutions of chaotic dynamics. Appl. Math. Mech. Engl. Ed. 2018, 39, 1529-1546. [CrossRef]

4. Lozi, R.; Pchelinstev, A.N. A new reliable numerical method for computing chaotic solutions of dynamical systems: The Chen attractor case. Int. J. Bifurc. Chaos 2015, 25, 1550187. [CrossRef] 
5. Haq, B.U.; Naeem, I. First integrals and analytical solutions of some dynamical systems. Nonlinear Dyn. 2019, 95, $1747-1765$. [CrossRef]

6. Mellodge, P. Chapter 4-Characteristics of Nonlinear Systems. In A Practical Approach to Dynamical Systems for Engineers; Mellodge, P., Ed.; Woodhead Publishing: Cambridge, UK, 2016; pp. 215-250. ISBN 9780081002025.

7. Ling, W.K. Nonlinear Digital Filters: Analysis and Applications; Academic Press: New York, NY, USA, $2010 ;$ ISBN 9780080550015.

8. Puy, A.; Daza, A.; Wagemakers, A.; Sanjuán, M.A.F. A test for fractal boundaries based on the basin entropy. Commun. Nonlinear Sci. Numer. Simul. 2021, 95, 105588. [CrossRef]

9. Goufo, E.F.D.; Khan, Y. A new auto-replication in systems of attractors with two and three merged basins of attraction via control. Commun. Nonlinear Sci. Numer. Simul. 2021, 96, 105709. [CrossRef]

10. Yan, Y.; Xu, J.; Wiercigroch, M.; Guo, Q. Statistical basin of attraction in time-delayed cutting dynamics: Modelling and computation. Phys. D Nonlinear Phenom. 2021, 416, 132779. [CrossRef]

11. Lee, M.Y.; Kim, Y.I. Development of a Family of Jarratt-Like Sixth-Order Iterative Methods for Solving Nonlinear Systems with their basins of attraction. Algorithms 2020, 13, 303. [CrossRef]

12. Rabenimananaa, T.; Walter, V.; Kacem, N.; Le Moal, P.; Bourbon, G.; Lardiès, J. Functionalization of electrostatic nonlinearities to overcome mode aliasing limitations in the sensitivity of mass microsensors based on energy localization editors-pick. Appl. Phys. Lett. 2020, 117, 033502. [CrossRef]

13. Ngo, G.Q.; George, A.; Schock, R.T.K.; Tuniz, A.; Najafidehaghani, E.; Gan, Z.; Geib, N.C.; Bucher, T.; Knopf, H.; Saravi, S.; et al Scalable functionalization of optical fibers using atomically thin semiconductors. Adv. Mater. 2020, 32, e2003826. [CrossRef]

14. Rajagopal, K.; Akgul, A.; Jafari, S.; Karthikeyan, A.; Cavusoglu, U.; Kacar, S. An exponential jerk system, its fractional-order form with dynamical analysis and engineering application. Soft Comput. 2020, 24, 7469-7479. [CrossRef]

15. Quan, G.Z.; Ma, Y.Y.; Zhang, Y.Q.; Zhang, P.; Wang, W.Y. Separation of dynamic recrystallization parameter domains from a chaotic system for Ti-6Al-4V alloy and its application in parameter loading path design. Mater. Sci. Eng. A 2020, $772,138745$. [CrossRef]

16. Changaival, B.; Rosalie, M.; Danoy, G.; Lavangnananda, K.; Bouvry, P. Chaotic Traversal (CHAT): Very Large Graphs Traversal Using Chaotic Dynamics. Int. J. Bifurc. Chaos 2017, 27, 1750215. [CrossRef]

17. Li, C.T.; Lee, C.C.; Weng, C.Y.; Chen, S.J. A Secure Dynamic Identity and Chaotic Maps Based User Authentication and Key Agreement Scheme for e-Healthcare Systems. J. Med. Syst. 2016, 40, 233. [CrossRef]

18. Zang, X.; Iqbal, S.; Zhu, Y.; Liu, X.; Zhao, J. Applications of Chaotic Dynamics in Robotics. Int. J. Adv. Robot. Syst. 2016, 13. [CrossRef]

19. Akgul, A.; Moroz, I.; Pehlivan, I.; Vaidyanathan, S. A new four-scroll chaotic attractor and its engineering applications. Optik 2016, 127, 5491-5499. [CrossRef]

20. Ott, E. Chaos in Dynamical Systems; Cambridge University Press: Cambridge, UK, 1993.

21. Din, Q. Qualitative analysis and chaos control in a density-dependent host-parasitoid system. Int. J. Dyn. Control 2018, 6, 778-798. [CrossRef]

22. Nobakhti, E.; Khaki-Sedigh, A.; Vasegh, N. Control of multichaotic systems using the extended OGY method. Int. J. Bifurc. Chaos 2015, 25, 1550096. [CrossRef]

23. Din, Q.; Elsadany, A.A.; Ibrahim, S. Bifurcation analysis and chaos control in a second order rational difference equation. Int. J. Nonlinear Sci. Numer. Simul. 2018, 19, 53-68. [CrossRef]

24. Leonov, G.A. Pyragas stabilizability via delayed feedback with periodic control gain. Syst. Control Lett. 2014, 69, 34-37. [CrossRef]

25. Xu, C.; Zhang, Q. On the chaos control of the Qi system. J. Eng. Math. 2015, 90, 67-81. [CrossRef]

26. Amster, P.; Alliera, C. Control of Pyragas applied to a coupled system with unstable periodic orbits. Bull. Math. Biol. 2018, 80, 2897-2916. [CrossRef] [PubMed]

27. Schwartz, I.B.; Triandaf, I.; Meucci, R.; Carr, T.W. Open-loop sustained chaos and control: A manifold approach. Phys. Rev. E 2002, 66, 026213. [CrossRef] [PubMed]

28. Li, Y.N.; Yang, Y.T.; Zhu, Z.M.; Zhang, C.L. Feed-forward slope compensated PFC for chaos control. J. Circuits Syst. Comput. 2015, 25, 1550065. [CrossRef]

29. Danca, M.-F.; Chattopadhyay, J. Chaos control of Hastings-Powell model by combining chaotic motions. Chaos 2016, 26, 043106. [CrossRef] [PubMed]

30. Danca, M.-F.; Garrappa, R.; Tang, W.K.S.; Chen, G. Sustaining stable dynamics of a fractional-order chaotic financial system by parameter switching. Comput. Math. Appl. 2013, 66, 702-716. [CrossRef]

31. Danca, M.-F.; Lung, N. Parameter switching in a generalized Duffing system: Finding the stable attractors. Appl. Math. Comput. 2013, 223, 101-114. [CrossRef]

32. Núñez-Pérez, J.C.; Adeyemi, V.A.; Sandoval-Ibarra, Y.; Pérez-Pinal, F.J.; Tlelo-Cuautle, E. FPGA Realization of Spherical Chaotic System with Application in Image Transmission. Math. Probl. Eng. 2021, 2021, 5532106. [CrossRef]

33. Tuna, M.; Alçın, M.; Koyuncu, I.; Fidan, C.B.; Pehlivan, I. High speed FPGA-based chaotic oscillator design. Microprocess. Microsystem. 2019, 66, 72-80. [CrossRef]

34. Yang, C.H.; Huang, S.J. Secure color image encryption algorithm based on chaotic signals and its FPGA realization. Int. J. Circuit Theory Appl. 2018, 46, 2444-2461. [CrossRef] 
35. Alçin, M.; Koyuncu, I.; Tuna, M.; Varan, M.; Pehlivan, I. A novel high speed artifical neural network-based chaotic true random number generator on field programmable gate array. Int. J. Circuit Theory Appl. 2018, 47, 365-378. [CrossRef]

36. Li, P.; Zhang, W.; Li, Z.; Liu, W.; Halang, W.A. FPGA implementation of a coupled-map-lattice-based cryptosystem. Int. J. Circuit Theory Appl. 2010, 38, 85-98. [CrossRef]

37. Tlelo-Cuautle, E.; de la Fraga, L.G.; Viet-Thanh, P.; Volos, C.; Jafari, S. Quintas-Valles, A.J. Dynamics, FPGA realization and application of a chaotic system with an infinite number of equilibrium points. Nonlinear Dyn. 2017, 89, 1129-1139. [CrossRef]

38. Yu, F.; Liu, L.; He, B.; Huang, Y.; Shi, C.; Cai, S.; Song, Y.; Du, S.; Wan, Q. Analysis and FPGA realization of a novel 5D hyperchaotic four-wing memristive system, active control synchronization, and secure communication application. Complexity 2019, 2019, 4047957. [CrossRef]

39. Azzaz, M.S.; Tanougast, C.; Maali, A.; Benssalah, M. An efficient and lightweight multi-scroll chaos-based hardware solution for protecting fingerprint biometric templates. Int. J. Commun. Syst. 2019, 33, e4211. [CrossRef]

40. Hagras, E.A.A.; Saber, M. Low power and high-speed FPGA implementation for 4D memristor chaotic system for image encryption. Multimed. Tools Appl. 2020, 79, 23203-23222. [CrossRef]

41. Guillén-Fernández, O.; Meléndez-Cano, A.; Tlelo-Cuautle, E.; Núñez-Pérez, J.C.; Rangel-Magdaleno, J.J. On the synchronization techniques of chaotic oscillators and their FPGA-based implementation for secure image transmission. PLoS ONE 2019, 14, e0209618. [CrossRef]

42. Tlelo-Cuautle, E.; Díaz-Muñoz, J.D.; González-Zapata, A.M.; Li, R.; León-Salas, W.D.; Fernández, F.V.; Guillén-Fernández, O.; Cruz-Vega, I. Chaotic Image Encryption Using Hopfield and Hindmarsh-Rose Neurons Implemented on FPGA. Sensors 2020, 20, 1326. [CrossRef] [PubMed]

43. Tlelo-Cuautle, E.; Pano-Azucena, A.D.; Guillén-Fernández, O.; Silva-Juárez, A. Synchronization and Applications of Fractional-Order Chaotic Systems. In Analog/Digital Implementation of Fractional Order Chaotic Circuits and Applications; Springer: Cham, Switzerland, 2019.

44. Sivaraman, R.; Rajagopalan, S.; Amirtharajan, R. FPGA based generic RO TRNG architecture for image confusion. Multimed. Tools Appl. 2020, 79, 13841-13868. [CrossRef]

45. Zeng, J.; Wang, C. A Novel Hyperchaotic Image Encryption System Based on Particle Swarm Optimization Algorithm and Cellular Automata. Secur. Commun. Netw. 2021, 2021, 6675565. [CrossRef]

46. Wang, X.; Li, Y.; Jin, J. A new one-dimensional chaotic system with applications in image encryption. Chaos Solitons Fractals 2020, 139, 110102.

47. Li, Z.; Peng, C.; Tan, W.; Li, L. An Efficient Plaintext-Related Chaotic Image Encryption Scheme Based on Compressive Sensing. Sensors 2021, 21, 758. [CrossRef] [PubMed]

48. Mohamed, A.G.; Korany, N.O.; El-Khamy, S. E. New DNA Coded Fuzzy Based (DNAFZ) S-Boxes: Application to Robust Image Encryption Using Hyper Chaotic Maps. IEEE Access 2021, 9, 14284-14305. [CrossRef]

49. Núñez Pérez, J.C.; Adeyemi, V.A.; Sandoval-Ibarra, Y.; Serrato-Andrade, R.Y.; Cárdenas, J.R.; Tlelo-Cuautle, E. Mathematical and numerical analysis of the dynamical behavior of Chen oscillator. Int. J. Dyn. Control 2020, 8, 386-395. [CrossRef]

50. Barboza, R. On the Lorenz and Chen Systems. Int. J. Bifurc. Chaos 2018, 28, 1850018. [CrossRef]

51. Chen, G.; Ueta, T. Yet another chaotic attractor. Int. J. Bifurc. Chaos 1999, 9, 1465-1466. [CrossRef]

52. Wang, X.; Chen, G. Generating Lorenz-like and Chen-like attractors from a simple algebraic structure. Sci. China Inf. Sci. 2014, 57, 1-7. [CrossRef]

53. Danca, M.-F.; Feckan, M. Note on a parameter-switching method for nonlinear ODEs. Math. Slovaca 2016, 66, 439-448. [CrossRef]

54. Tang, W.K.S.; Danca, M.-F. Emulating "Chaos + Chaos = Order" in Chen's circuit of fractional order by parameter switching. Int. J. Bifurc. Chaos 2016, 26, 1650096. [CrossRef]

55. Danca, M.-F. Convergence of a parameter switching algorithm for a class of nonlinear continuous systems and a generalization of Parrondo's paradox. Commun. Nonlinear Sci. Numer. Simul. 2013, 18, 500-510. [CrossRef]

56. Sira-Ramirez, H.; Cruz-Hernandez, C. Synchronization of chaotic systems: A generalized Hamiltonian systems approach. Int. J. Bifurc. Chaos 2001, 11, 1381-1395. [CrossRef]

57. Pei, L.J.; Liu, S.H. Application of generalized Hamiltonian systems to chaotic synchronization. Nonlinear Dyn. Syst. Theory 2009, 9, 415-432.

58. Danca, M.-F.; Tang, W.K.S. Parrondo's paradox for chaos control and anticontrol of fractional-order systems. Chin. Phys. B 2016, 25, 010505. [CrossRef]

59. Harmer, G.P.; Abbott, D. A review of Parrondo's paradox. Fluct. Noise Lett. 2002, 2, R71-R107. [CrossRef]

60. Kumar, V.; Aggarwal, R.; Sharma, P.; Kaur, B. Fractal basins of attraction in a binary quasar model. New Astron. 2021, 84, 101543. [CrossRef]

61. Saeed, T.; Chen, W.; Zotos, E.E. Convergence properties of equilibria in the restricted three-body problem with prolate primaries. Astron. Nachrichten 2020, 341, 887-898. [CrossRef]

62. Koppu, S.; Viswanatham, V.M. A fast enhanced secure image chaotic cryptosystem based on hybrid chaotic magic transform. Model. Simul. Eng. 2017, 2017, 7470204. [CrossRef]

63. Chang, D.; Li, Z.; Wang, M.; Zeng, Y. A novel digital programmable multi-scroll chaotic system and its application in FPGA-based audio secure communication. AEU Int. J. Electron. Commun. 2018, 88, 20-29. [CrossRef] 
64. Tlelo-Cuautle, E.; Carbajal-Gomez, V.H.; Obeso-Rodelo, P.J.; Rangel-Magdaleno, J.J.; Núñez-Pérez, J.C. FPGA realization of a chaotic communication system applied to image processing. Nonlinear Dyn. 2015, 82, 1879-1892. [CrossRef]

65. Sadoudi, S.; Tanougast, C.; Azzaz, M.S.; Dandache, A. Design and FPGA implementation of a wireless hyperchaotic communication system for secure real-time image transmission. EURASIP J. Image Video Process. 2013, 43. [CrossRef]

66. Belo, D.; Carvalho, N.B. An OOK chirp spread spectrum backscatter communication system for wireless power transfer applications. IEEE Trans. Microw. Theory Tech. 2021, 69, 1838-1845. [CrossRef]

67. Liu, M.; Han, Y.; Chen, Y.; Song, H.; Yang, Z.; Gong, F. Modulation parameter estimation of LFM interference for direct sequence spread spectrum communication system in Alpha-Stable noise. IEEE Syst. J. 2021, 15, 881-892. [CrossRef]

68. Sheikhpour, S.; Mahani, A.; Bagheri, N. Reliable advanced encryption standard hardware implementation: 32-bit and 64-bit data-paths. Microprocess. Microsystem. 2021, 81, 103740. [CrossRef]

69. Ikhwan, A.; Rafikha Aliana, A.R.; Ehkan, P.; Yacob, Y.; Syaifuddin, M. Data Security Implementation using Data Encryption Standard Method for Student Values at the Faculty of Medicine, University of North Sumatra. J. Phys. Conf. Ser. 2021, 1755, 012022. [CrossRef] 